\title{
SUPPLEMENTARY MATERIAL FOR: A GENERAL THEORY FOR PREFERENTIAL SAMPLING IN ENVIRONMENTAL NETWORKS
}

\author{
By Joe Watson ${ }^{1 \S}$ And James V ZideK ${ }^{1 \S}$ And Gavin Shaddick ${ }^{\dagger}$ \\ University of British Columbia ${ }^{\S}$ and University of British Columbia ${ }^{\dagger}$ and University of Exeter $\ddagger$
}

\section{Supplementary Material.}

1.1. Chosen priors for the case study. For the $Y$ process, we used weakly informative Gaussian priors for the $\gamma_{k}$ 's. We used a $\operatorname{Gamma}(a, b)$ prior for the precision parameter $1 / \sigma_{\epsilon}^{2}$, where $a$ denotes the shape parameter and $b$ denotes the inverse-scale parameter. We chose $a=1$ and $b=5 \times 10^{-} 5$. Under this parameterisation, the mean and variance of this distribution are $a / b$ and $a / b^{2}$ respectively. Thus this prior assumption allows for very large and very small variances of the response to exist. Next, the a $2 \mathrm{D}$ Wishart distribution is assumed for $\Sigma_{b}^{-1}$ with four degrees of freedom. The prior matrix is given 0 off-diagonal elements and diagonal values of 1 . This results in a prior mean for the two variance terms of the random effects $\left(\sigma_{b, 1}^{2}, \sigma_{b, 2}^{2}\right)$ of 4 with a prior variance for these terms equal to 8 . The prior mean for the correlation term is 0 with variance for the logit transform of the correlation equal to 4 . This allows for random effects with a large range of magnitudes and correlation structures to exist. We place the PC joint priors (??) on the two hyperparameters for the 3 independent Matern realisations, with prior belief that the lower 5 th percentile for the range is $3.4 \mathrm{~km}$ (a fifth of the smallest range found in previous analyses) and the upper 1st percentile for the standard deviation of each field is 1 (noting that the data have been transformed). We fix the Matern roughness parameter to equal 1 since this is the largest smoothness value currently implemented in R-INLA, and we assume a-priori that the medium-range pollution process will be reasonably smooth. The lower prior bound on the range parameter, combined with the probabilistic upper bound on the variance, should help prevent the model from collapsing into a state that over-fits the data.

For the site-selection process $R$, our choice of priors follows the same objectives as for the observation process. Weakly informative Gaussian priors were placed on all the $\alpha$ terms. The same PC prior chosen for the observation process was placed on the $\beta_{0}^{\star}(\mathbf{s})$ field. For the first order autoregressive term $\beta_{1}^{\star}(t)$, we placed a Gamma $\left(1,5 \times 10^{-} 5\right)$ on the marginal precision and a $N(0,0.15)$ prior was placed on the logit of the lag 1 correlation (i.e. on $\left.\log \left(\left(1+\rho_{a}\right) /\left(1-\rho_{a}\right)\right)\right)$ to allow for a large degree of flexibility. Finally, we consider two different sets of priors for the PS parameters $d_{b}, d_{\beta}$. For Implementation 1 we constrain these to equal 0 and thus we can view this as setting a point mass prior at 0 . For implementations 2 and 3 , we assign a $N(0,10)$ prior to allow PS to be detected.

1.2. Details on the R-INLA implementation. We used the estimated ranges from ? to construct the Delauney triangulation mesh required for use in R-INLA. Following the advice of ??,

\footnotetext{
$\S$ The research reported in this article was partially supported by the Natural Science and Engineering Research Council of Canada.

AMS 2000 subject classifications: Primary 62P12; secondary 62D99

Keywords and phrases: preferential sampling, INLA, random fields, mobile monitors, health effects, air pollution, Big Data
} 
and trading it off with the need for maintaining a reasonable computation time, we set the edge lengths of the triangles throughout the domain to be around $5 \mathrm{~km}$, less than the minimum estimated range of $17 \mathrm{~km}$ found in ?. This is important since it has been shown that the length of the triangle edges must be less than the range of any Matern field and should ideally be less than a quarter of this. Failure to do so leads to large errors in the approximation of the Gaussian random field. We are confident that with our choice of mesh, any changes to the inference in the unsampled regions will be a direct result of our joint model framework and not due to any undesirable artifacts caused by a poor choice of triangulation mesh for the SPDE approximation.

It is well known that an empirical Bayes or maximum likelihood approach does not fully account for the uncertainties in the hyperparameters when performing predictions and inference, and these may be high in spatially correlated Gaussian random fields (?). Interestingly, for this dataset we compared the fully Bayesian approach with the empirical Bayes method using R-INLA and found little difference. The posterior credible intervals for the latent effects and parameters were slightly wider under the fully Bayesian approach, however the posterior credible intervals for the predictions were almost identical. Additionally we used the empirical Bayes approach in a small simulation study with good results. Thus for computational savings we opted to consider only empirical Bayes methods.

In R-INLA, copying across a linear combination of latent processes (potentially from a different time point) requires the use of dummy variables. In particular, the idea of ? is required. This simply involves creating infinite precision Gaussian variables with observed values of zero and with linear predictor set equal to the (negative) linear combination of latent processes desired, plus an infinite variance random intercept process. It is not hard to see that the values of these random intercepts equal precisely the values of the linear combination of the desired processes. This approach proved vital for fitting implementations 2 and 3.

Note that in essence, for Implementation 3 we are modelling the initial site-placement process as a LGCP, but using a Bernoulli likelihood as a pseudo-likelihood instead of the usual Poisson likelihood to form the computational approximation. We use the conditional logistic regression approach, commonly used to fit Poisson point processes, placing the zeros in a regular (not a latticed) manner throughout $\mathcal{S}$, independent from the observed site locations. In practice, we created a reasonably regular delauney triangulation mesh in R-INLA throughout $\mathcal{S}$ for our GMRF with mesh vertices placed independent from the observed site locations. Regularity was enforced through a combination of the choices of a minimum vertex length of $5 \mathrm{~km}$, an upper vertex length of $7 \mathrm{~km}$ and a minimum angle of 25 degrees. We then used the created mesh vertices as our pseudo-sites.

A somewhat undesirable property of using the logistic regression approach is that the likelihood value does not converge as the number of pseudo zeros tends towards infinity. Thus, unlike the result of using the Poisson approximation to a Point Process, convergence must instead be judged with the convergence of fixed parameter estimates, excluding the estimate of the intercept. However, if the Poisson approximation is chosen, then it cannot be used to simultaneously model the retention process alongside the site-placement process and hence a third Bernoulli likelihood modeling the retention-process would be required. Thus in either case, there is a trade-off. Given that the computational time required to fit the model in R-INLA using the SPDE approach is affected more by the resolution of the computational mesh than by the number of observations, we can increase the density of the pseudo-sites with a reasonably small effect on the total computation time.

Thus for fitting Implementation 3, we follow the advice given in the literature (??). We 
repeatedly re-fit the joint model on an ever-increasing density of pseudo-sites until the parameters and predictions converge. We found that all estimates, except of course the site-selection intercept, stabilised once the average distance between pseudo-sites was decreased to $5 \mathrm{~km}$. This supports the claim that our estimates from our model are close to those of the joint triple model with a LGCP for the site-selection process, a Bernoulli likelihood for the site-retention process, and a Gaussian process for the observation process.

The correct placement of the zeros in the site-selection process is vital for the asymptotic convergence of the pseudo-likelihood to the LGCP. In particular, the asymptotics of the conditional logistic regression approximation used in our example with the logit link are only established when the zeros are either a realisation of a homogeneous Poisson point process, independent of the monitoring site locations (?), or when they are placed uniformly throughout the domain $\mathcal{S}$ (?). In either case, the density of the zeros must be uniform (at least in probability) throughout $\mathcal{S}$ for each year $j \in\{1, \ldots, 31\}$ and be placed independent from the observation locations.

A direct consequence of this is that for our site-selection process, we should not consider for selection at time $j$ the subset of observed sites (i.e. the subset of Population 1) that are offline at year $j$ (i.e. $S_{t_{j}}^{C}$ ). Put differently, we should not include the $R_{i, j}$ 's in Population 1 in the likelihood such that $r_{i, j}=0$. Erroneously doing so would lead to an increased density of zeros in the heavily sampled regions and thus a 'preferential sample' of zeros. Similarly, for the site-retention process at time $j$, we should only consider the sites online at the previous time $j-1$.

Putting these two processes together, the only zeros that should contribute to the joint Bernoulli likelihood at time $j$ are the pseudo-sites and the sites that were online at the previous time $j-1$ and were removed from the network at time $j$. In fact, we tested the sensitivity of the results to the above, re-fitting the model once by following the advice given above, and again but ignoring the advice and considering all the observed sites (operational and offline) for selection at each time step $j$, along with the pseudo-sites. Despite the former being more appropriate, we found no differences in estimates, but we required a higher density of pseudo-sites, and hence an increased computational cost to reduce this bias in the parameter estimates. This advice is therefore of most importance for the modelling of very large datasets where the number of unique observed site locations through time could be much higher than seen here.

To form all of our predictions and maps, we simulated 1000 MCMC samples of all the parameters and latent effects from the fitted models. This feature is available in the R-INLA package (???), by simply saving all the configuration settings generated by the software required to fit the model. We then formed all the site-specific trajectories by appropriately combining all latent effects and parameters in the linear predictor. We take the mean, the empirical upper $97.5 \%$ and empirical lower $2.5 \%$ values of the 1000 linear predictor estimates to form our credible intervals. Finally, to obtain the map of the pointwise expectations of the predictive distribution across GB, we used the MCMC samples of the latent effects and parameters (minus the IID site-specific effects) and linearly interpolated the estimated field throughout $\mathcal{S}$ on a regular lattice grid covering the the map of GB, before taking the empirical mean and standard deviation across the 1000 maps. To compute the average BS across the Whole GB, we take the mean (averaging across the pixels) of each the 1000 sampled/realised maps. Then, we take the mean, the empirical $2.5 \%$ and the empirical $97.5 \%$ values of these 1000 (mean) values.

\subsection{Posterior pointwise mean and pointwise standard deviation plots.}



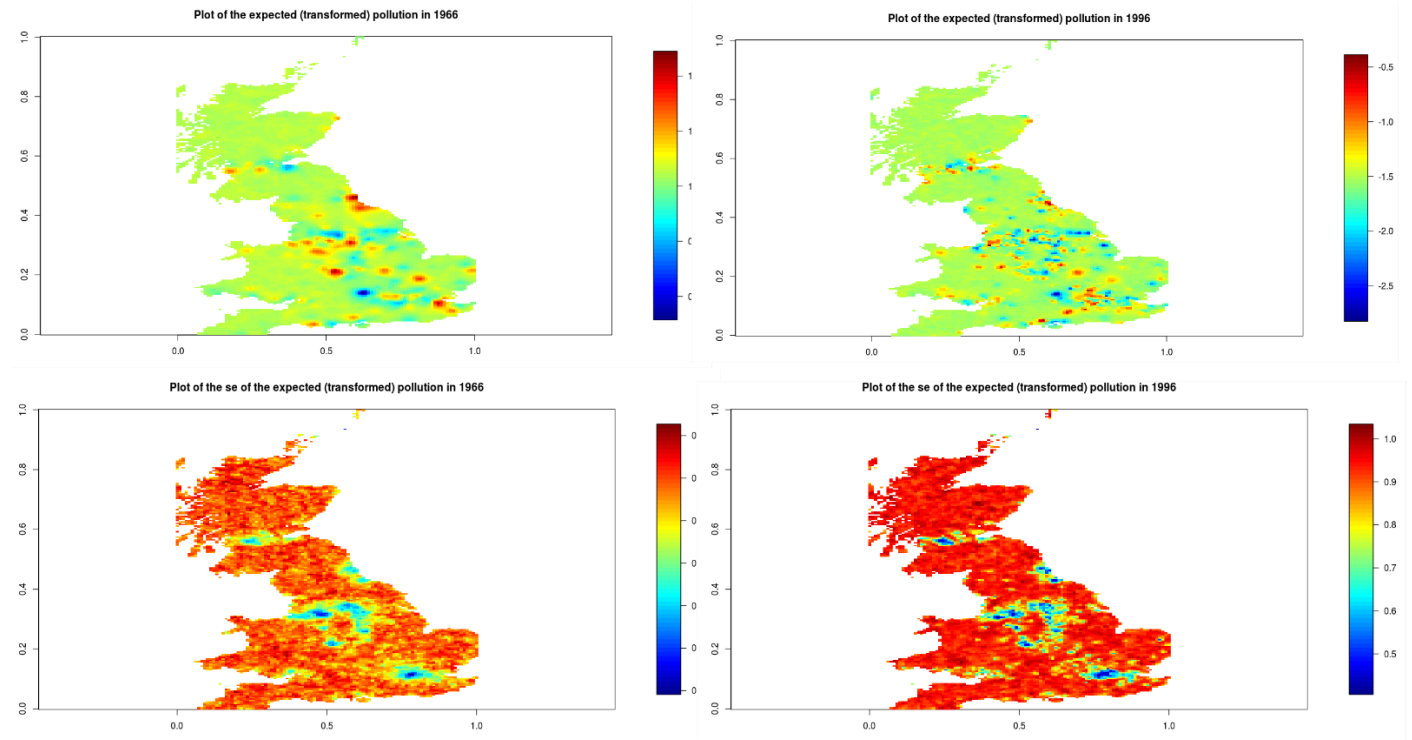

Fig 1: A plot of the posterior mean black smoke in 1966 and 1996 under Implementation 1 with corresponding standard errors plotted below. Note that for visualisation purposes, the two plots have had their values scaled to put them on the same colour scale.
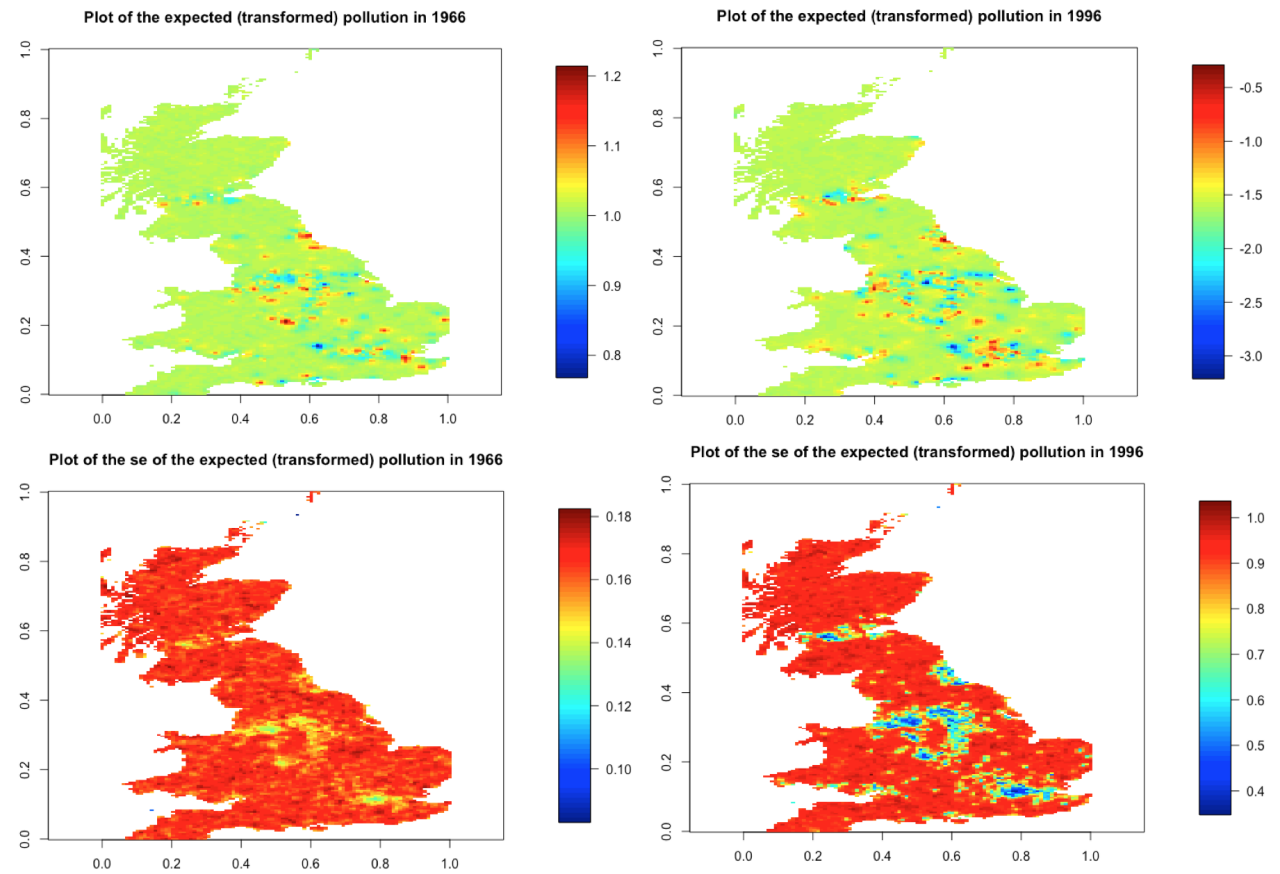

Fig 2: A plot of the posterior mean black smoke in 1966 and 1996 with corresponding standard errors plotted below. Estimates are taken from Implementation 2. Note that for visualisation purposes, the two plots have had their values scaled to put them on the same colour scale. 

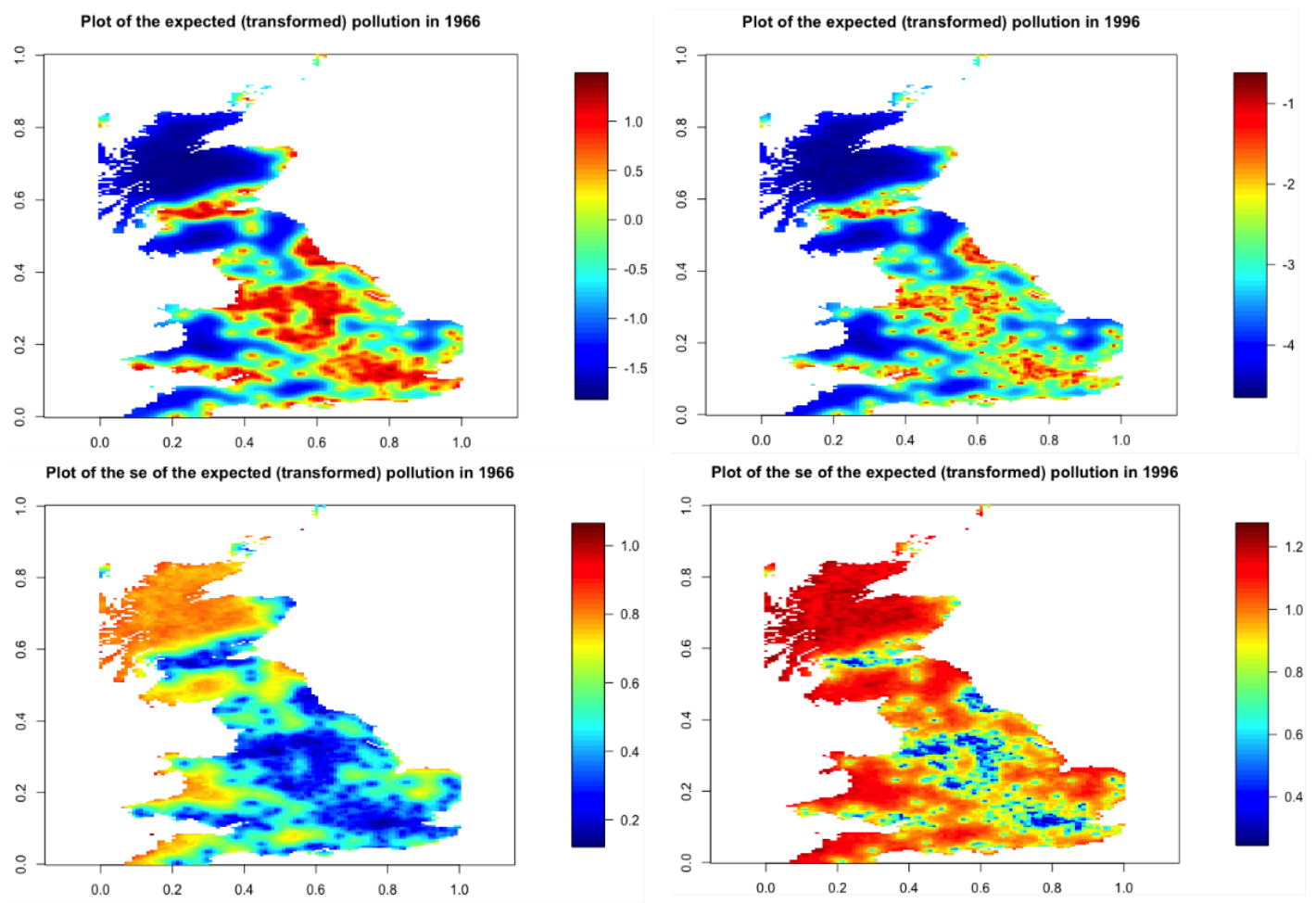

Fig 3: A plot of the posterior mean black smoke in 1966 and 1996 with corresponding standard errors plotted below. Estimates are taken from Implementation 3. Note that for visualisation purposes, the two plots have had their values scaled to put them on the same colour scale.

1.4. Additional plot of the exceedance of the annual black smoke EU guide value. 


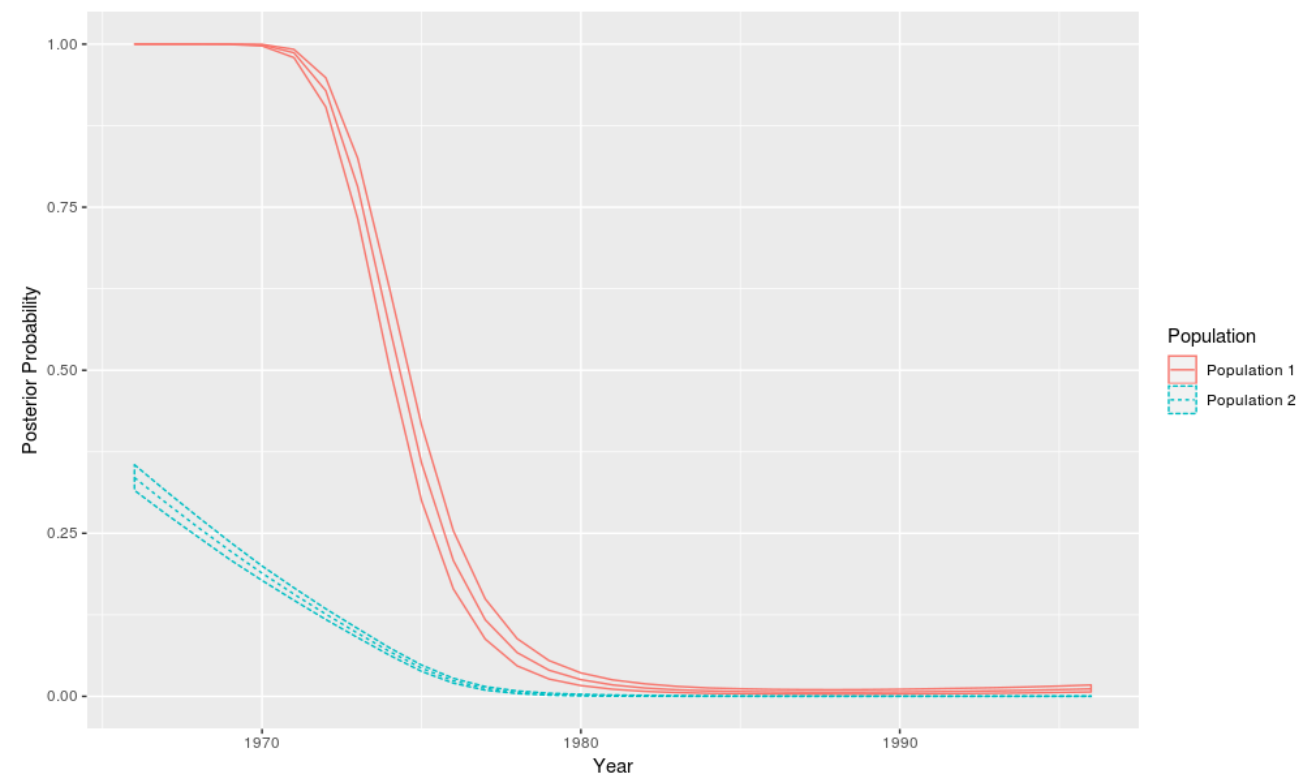

Fig 4: A plot showing the posterior proportion of the total surface area of Great Britain with annual average black smoke level exceeding the EU guide value of $34 \mu \mathrm{gm}^{-3}$. Shown are the results from Implementation 2 (the red solid line) and Implementation 3 (the blue dashed line). Note that the line for Implementation 1 is almost identical to that from Implementation 1 and omitted.

1.5. Additional plot of annual average black smoke levels. 


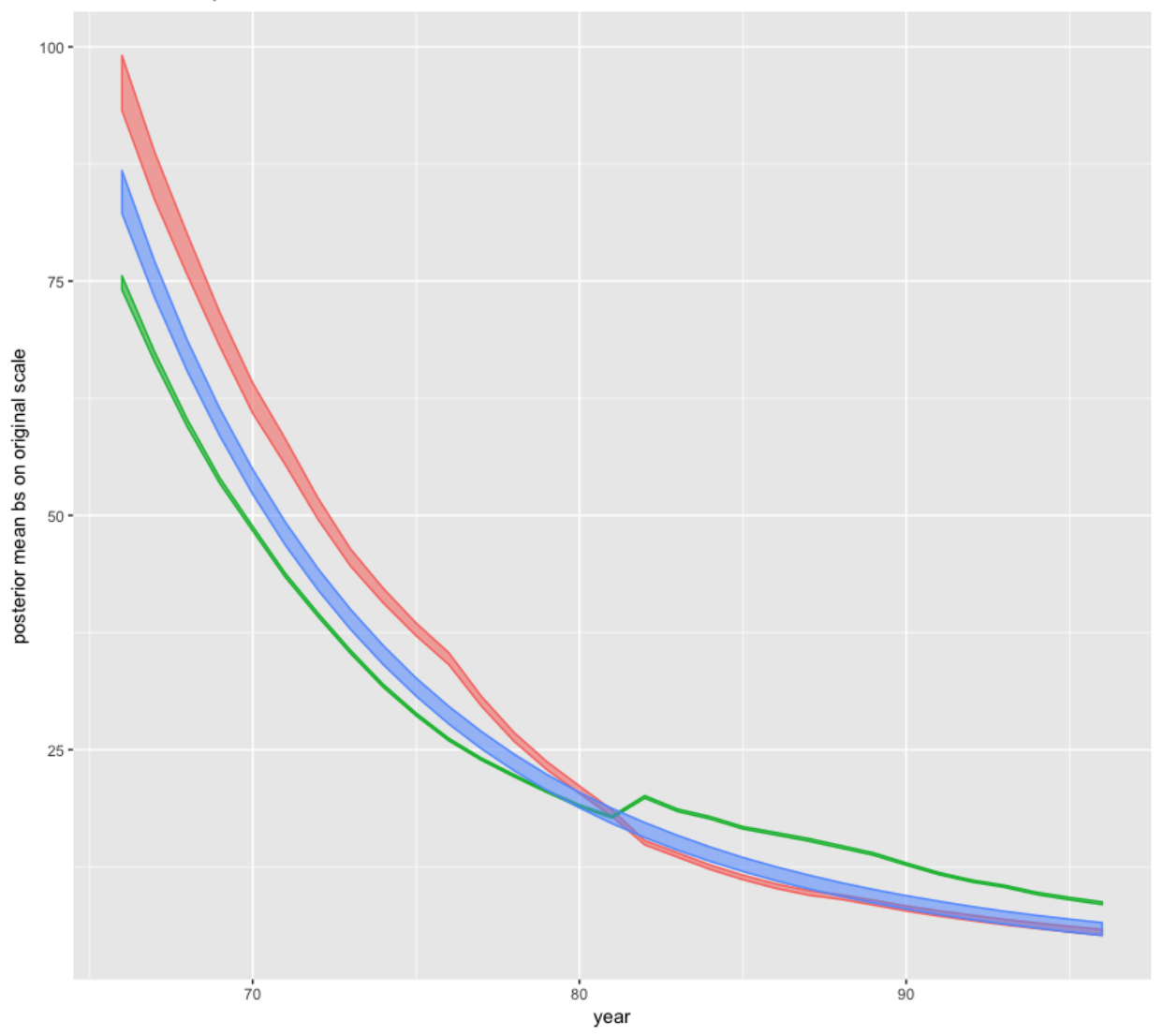

Fig 5: Implementation 2. In green are the BS levels averaged over sites that were selected in $\mathcal{P}_{1}$ (i.e. operational) at time t. In contrast, those in red are the BS levels averaged over sites that were not selected in $\mathcal{P}_{1}$ (i.e. offline) at time t. Finally, in blue are the BS levels averaged across Great Britain. Also included with the posterior mean values are their $95 \%$ posterior credible intervals. If printed in black-and-white, the green band is initially the lower line, the red band is the upper line and the blue band is initially the middle line.

1.6. Model diagnostic plots. We include, for each of the three implementations considered in this paper, residual plots to help diagnose poor model fit. Included are residuals vs. year plots, with a fitted lowess smoother to help show that the choice of a quadratic model adequately captured the temporal trend in the data. Also shown are normal QQ-plots of the residuals with fitted $99 \%$ confidence bands around the overlain QQ-line. It is clear from this plot that a heavier tailed distribution on the response would have been more suitable. Finally, we include histograms and normal QQ-plots of the random effects. Here we see slightly left-skewed and right-skewed empirical marginal distributions for the random intercepts and slopes respectively, however strictly speaking we should consider the empirical joint distribution of these effects. We have no strong cause for concern with these final plots. 


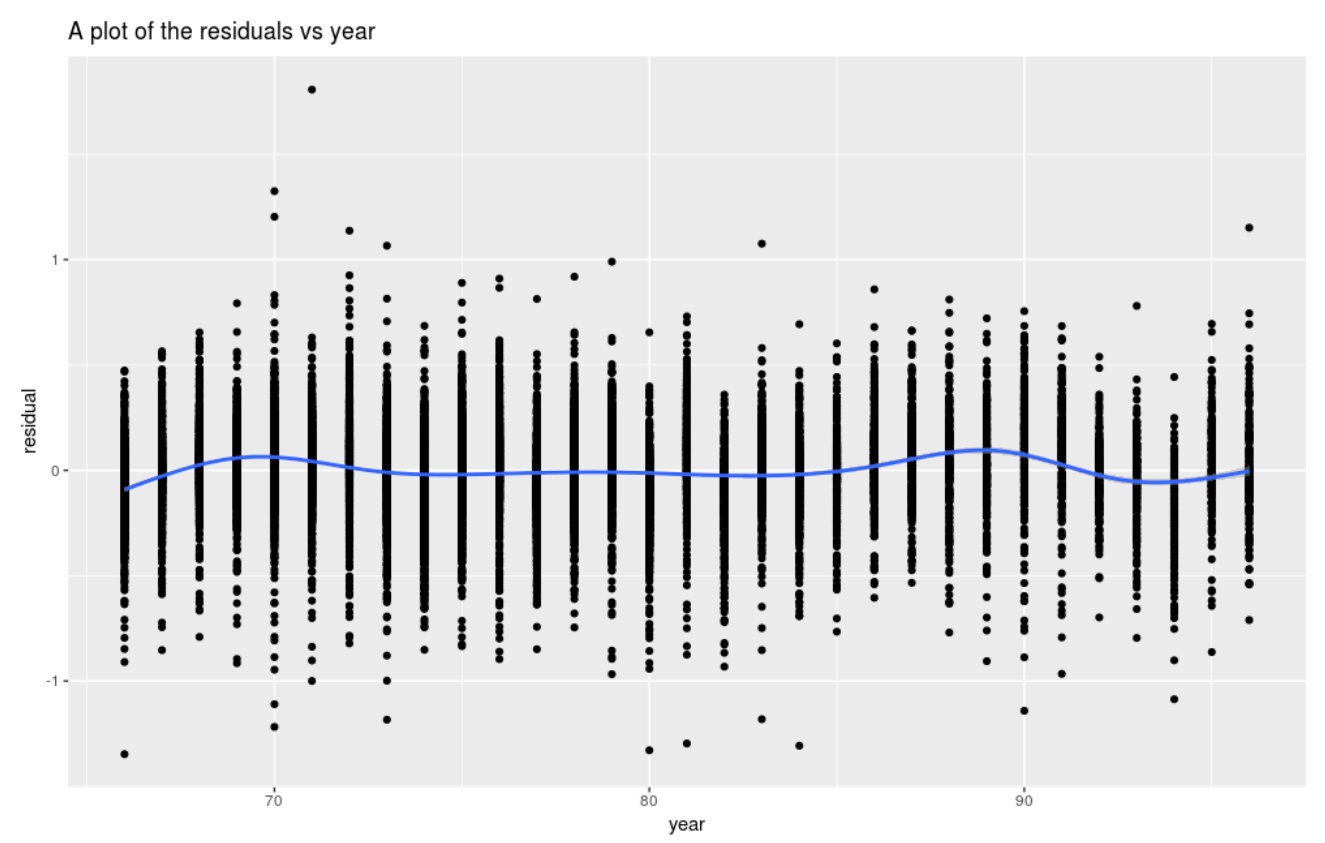

Fig 6: A plot of the residuals vs. year from Implementation 1 with a fitted smoother.

\section{Normal QQ-plot of residuals}

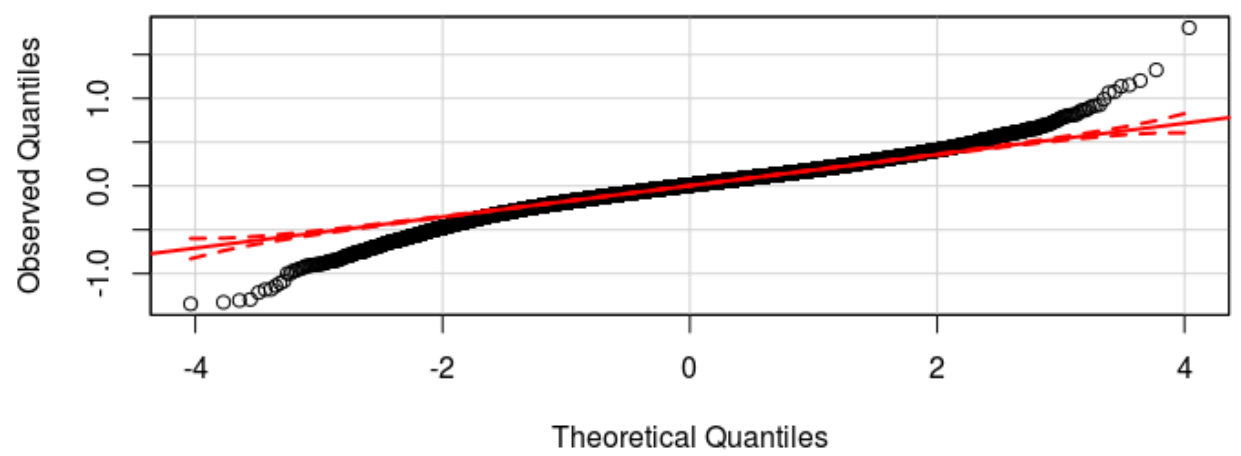

Fig 7: A Normal Q-Q plot of the residuals from Implementation 1. 
Histogram of random site-specific intercepts

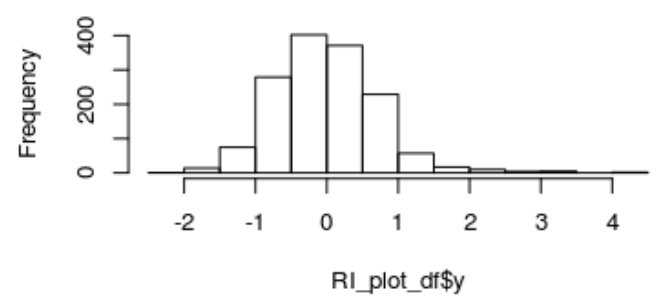

Histogram of random site-specific slopes

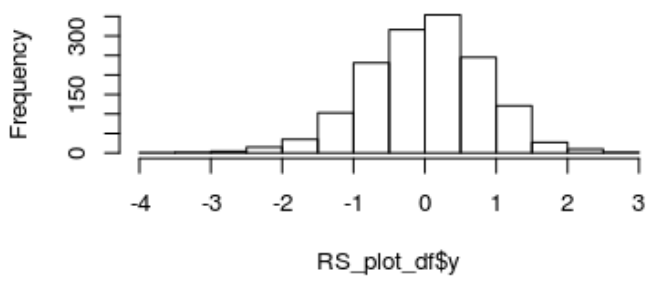

Normal Q-Q Plot

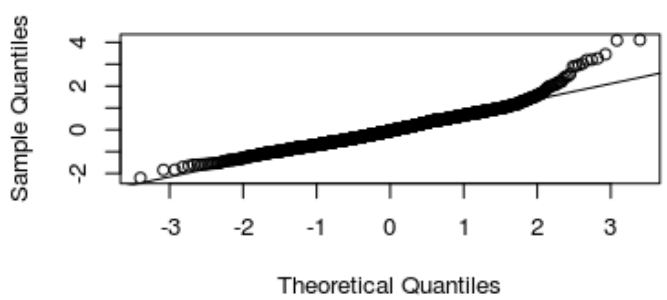

Normal Q-Q Plot

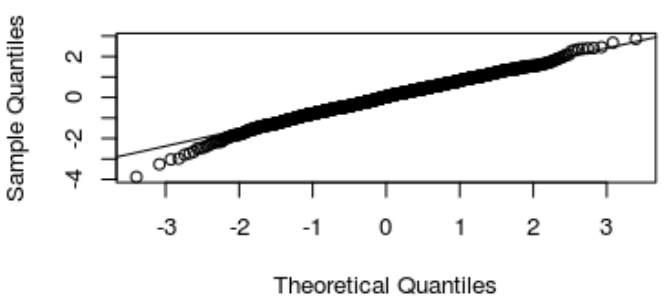

Fig 8: Histograms of the spatially-uncorrelated random intercepts (top left) and slopes(bottom left), with corresponding Normal Q-Q plots shown on the right from Implementation 1. 


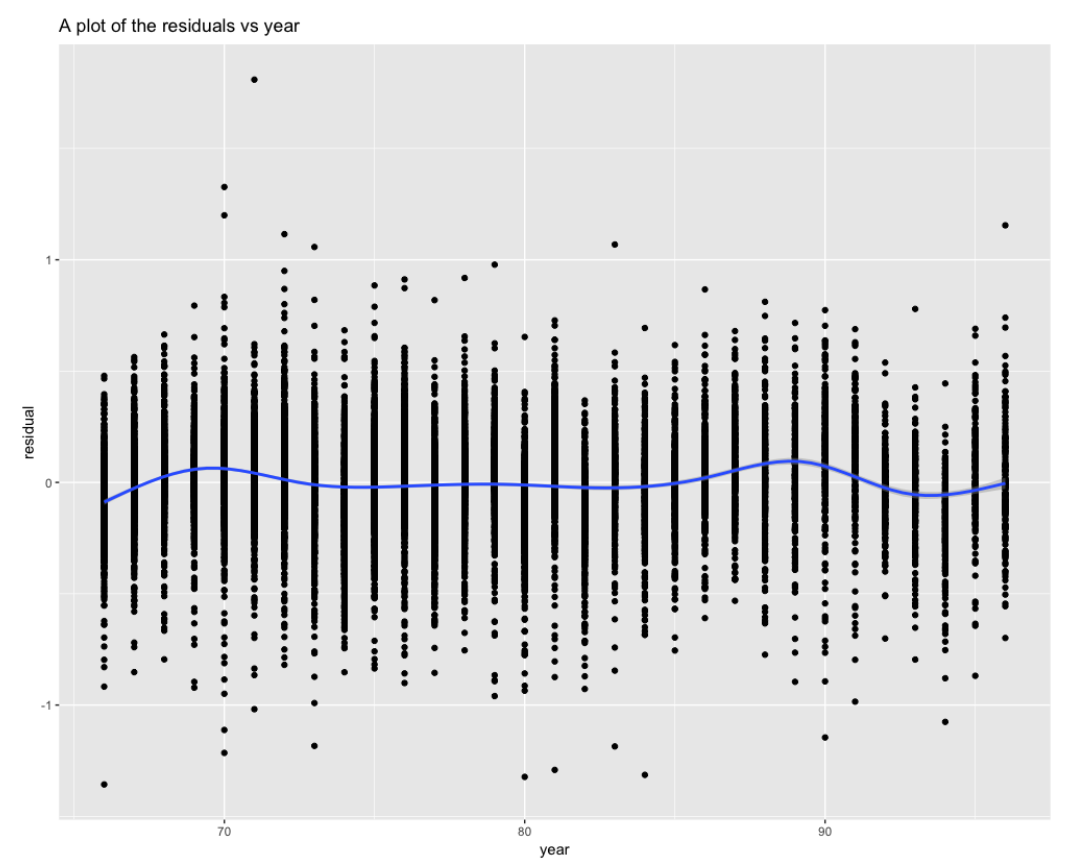

Fig 9: A plot of the residuals vs. year for Implementation 2, with a fitted smoother.

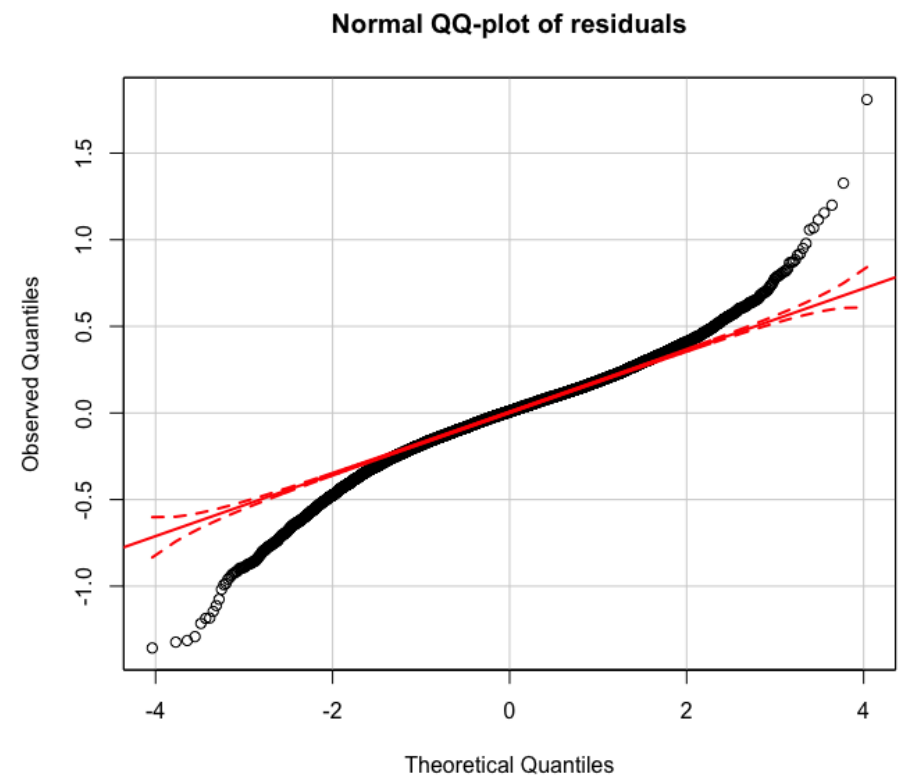

Fig 10: A Normal Q-Q plot of the residuals from Implementation 2, with 95\% confidence intervals shown in red. 
Histogram of random site-specific intercepts

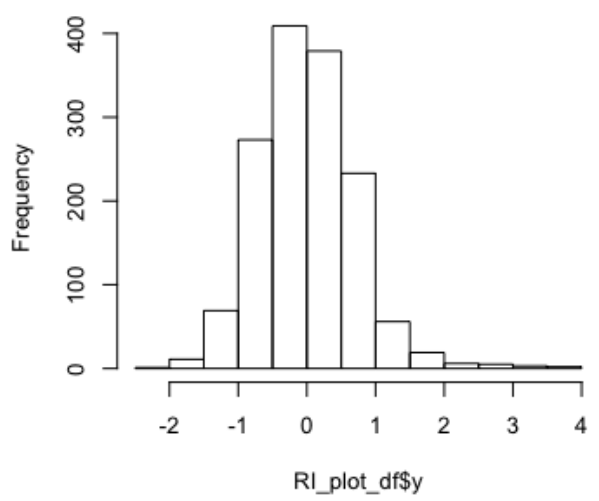

Histogram of random site-specific slopes

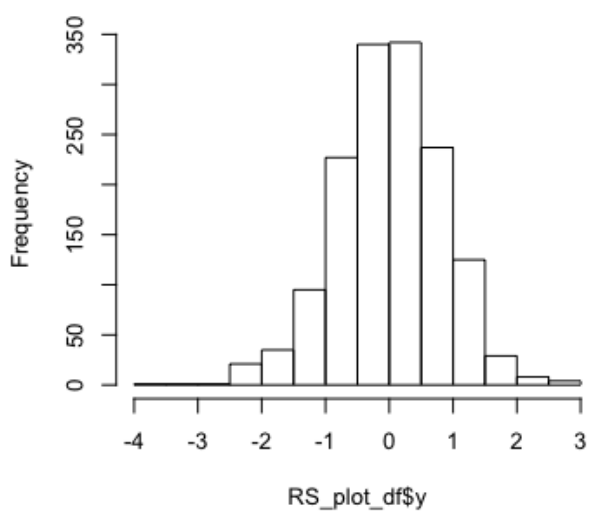

Normal Q-Q Plot

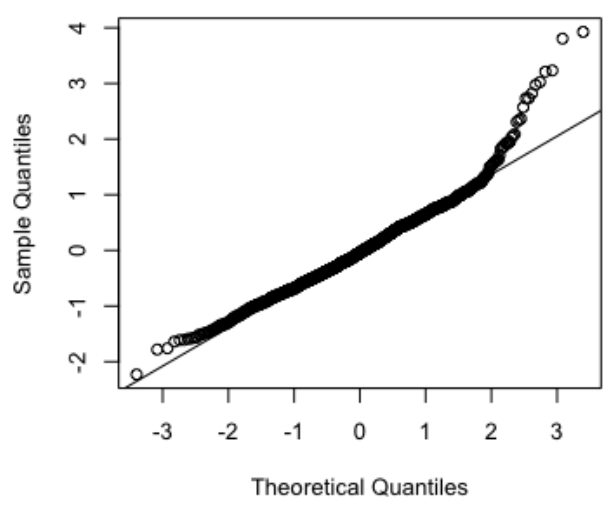

Normal Q-Q Plot

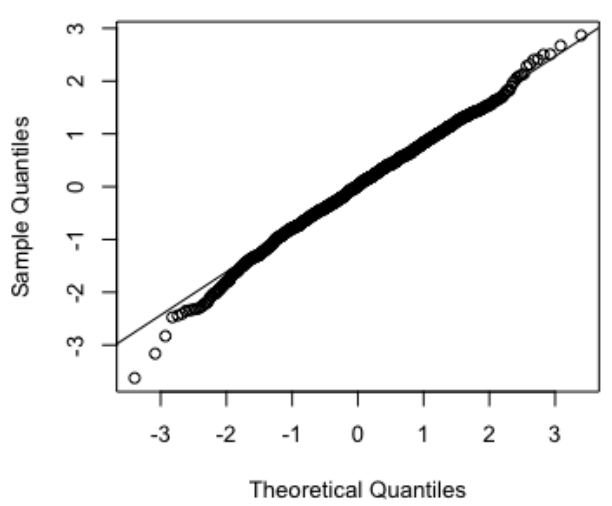

Fig 11: Histograms of the spatially-uncorrelated random intercepts (top left) and slopes(bottom left), with corresponding Normal Q- Q plots shown on the right from Implementation 2. 


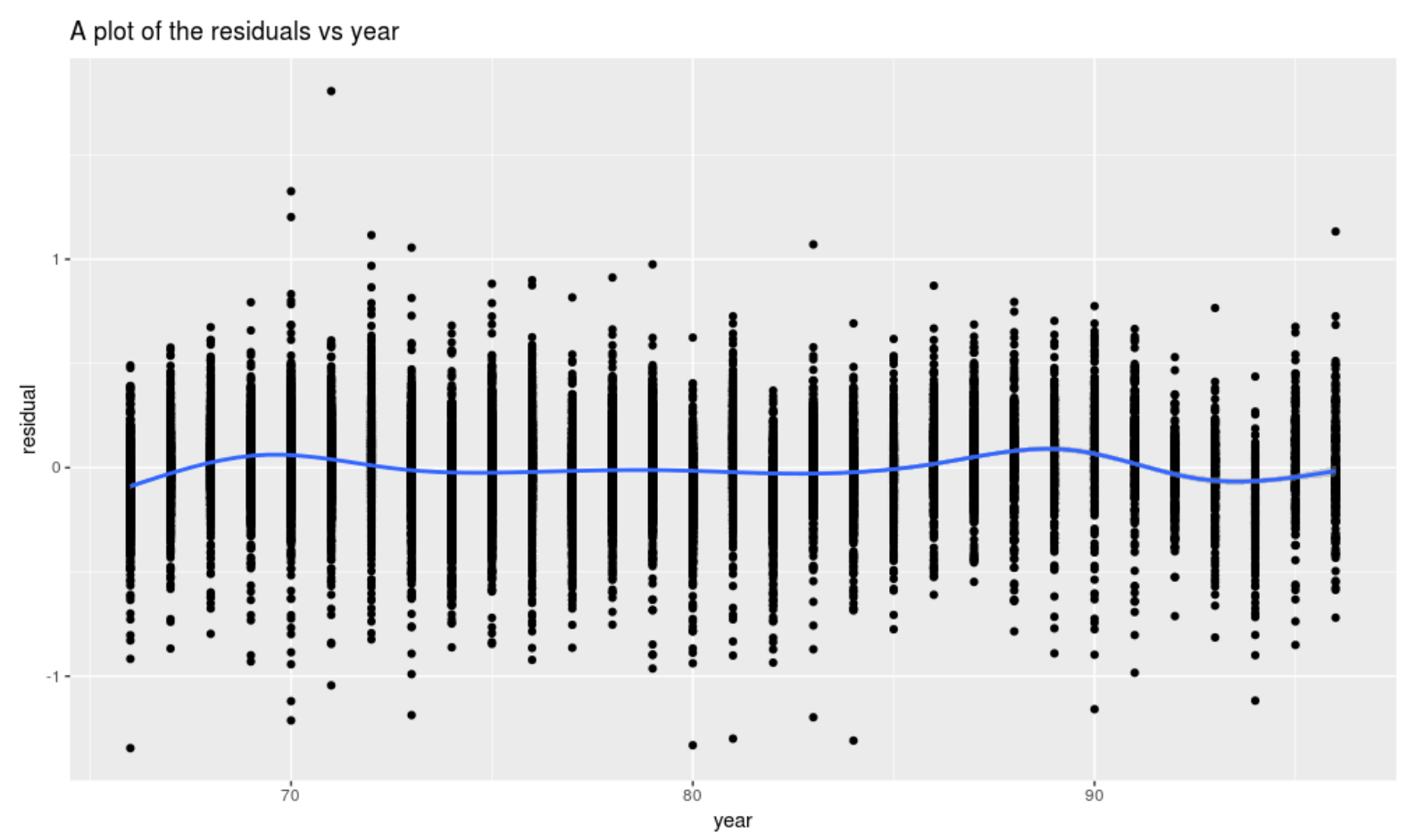

Fig 12: A plot of the residuals vs. year for Implementation 3 with a fitted smoother.

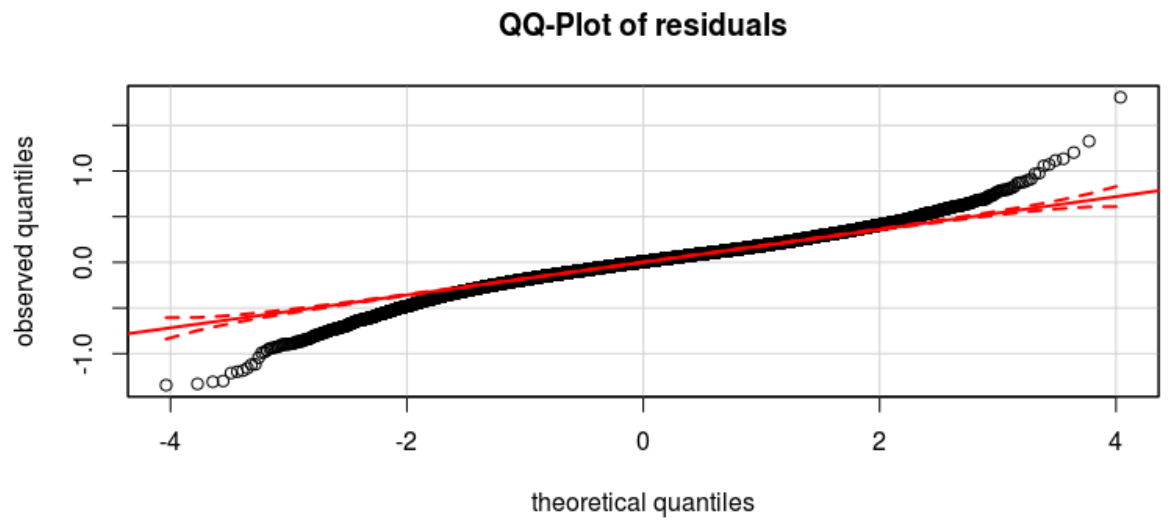

Fig 13: A Normal Q-Q plot of the residuals from Implementation 3 with 95\% confidence intervals shown in red. 


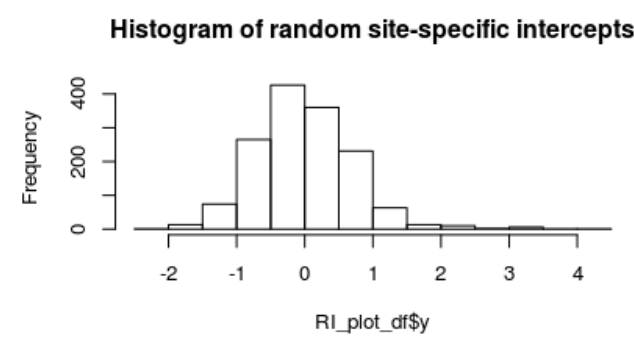

Histogram of random site-specific slopes

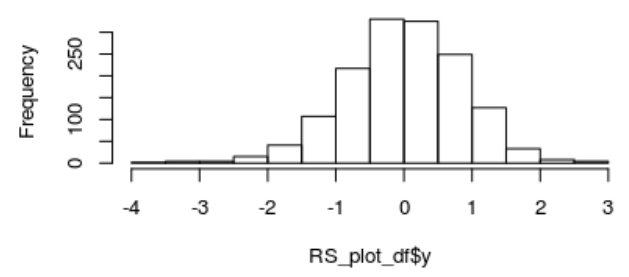

Normal Q-Q Plot

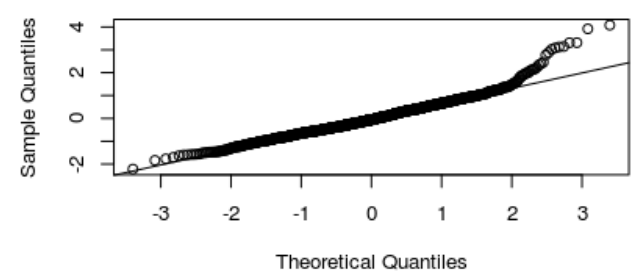

Normal Q-Q Plot

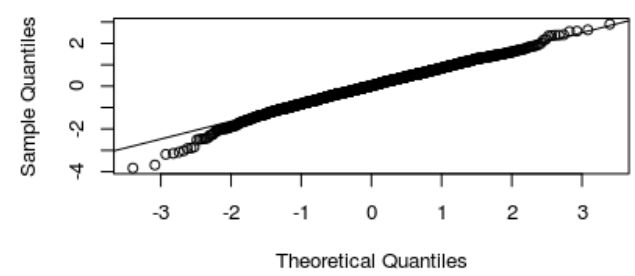

Fig 14: Histograms of the spatially-uncorrelated random intercepts (top left) and slopes(bottom right), with corresponding Normal Q-Q plots shown on the right from Implementation 3.

\section{References.}

Adrian Baddeley, Ege Rubak, and Rolf Turner. Spatial point patterns: methodology and applications with $R$. Chapman and Hall/CRC, 2015.

Haakon Bakka. Mesh creation including coastlines, Jan 2017. URL https://haakonbakka.bitbucket.io/ btopic104.html\#what-is-needed-for-a-good-mesh.

William Fithian and Trevor Hastie. Finite-sample equivalence in statistical models for presence-only data. The annals of applied statistics, 7(4):1917, 2013.

Geir-Arne Fuglstad, Daniel Simpson, Finn Lindgren, and Håvard Rue. Constructing priors that penalize the complexity of gaussian random fields. Journal of the American Statistical Association, 114(525):445-452, 2019.

Finn Lindgren, Håvard Rue, and Johan Lindström. An explicit link between gaussian fields and gaussian markov random fields: the stochastic partial differential equation approach. Journal of the Royal Statistical Society: Series B (Statistical Methodology), 73(4):423-498, 2011.

H. Rue, S. Martino, and N. Chopin. Approximate Bayesian inference for latent Gaussian models by using integrated nested Laplace approximations. Journal of the Royal Statistical Society: Series B (Statistical Methodology), 71 (2):319-392, 2009.

Håvard Rue, Andrea Riebler, Sigrunn H Sørbye, Janine B Illian, Daniel P Simpson, and Finn K Lindgren. Bayesian computing with inla: a review. Annual Review of Statistics and Its Application, 4:395-421, 2017.

Ramiro Ruiz-Cárdenas, Elias T Krainski, and Håvard Rue. Direct fitting of dynamic models using integrated nested laplace approximations-inla. Computational Statistics \& Data Analysis, 56(6):1808-1828, 2012.

Gavin Shaddick and James V Zidek. A case study in preferential sampling: Long term monitoring of air pollution in the uk. Spatial Statistics, 9:51-65, 2014.

Daniel Simpson, Håvard Rue, Andrea Riebler, Thiago G Martins, Sigrunn H Sørbye, et al. Penalising model component complexity: A principled, practical approach to constructing priors. Statistical science, 32(1):1-28, 2017.

David I Warton, Leah C Shepherd, et al. Poisson point process models solve the "pseudo-absence problem" for presence-only data in ecology. The Annals of Applied Statistics, 4(3):1383-1402, 2010. 
H. Zhang. Inconsistent estimation and asymptotically equal interpolations in model-based geostatistics. Journal of the American Statistical Association, 99(465):250-261, 2004. ISSN 0162-1459.

Sha Zhe. Mesh creation including coastlines, Aug 2017. URL http://rstudio-pubs-static.s3.amazonaws.com/ 302639_3ccd091c277d4d6c9ad9c2cf524250b6.html.

Department of Statistics

UNIVERSity of British COlumbia

2207 Main Mall Vancouver, BC

V6T 1Z4, CANADA

E-MAIL: joe.watson@stat.ubc.ca
Department of Statistics

University of British COlumbia

2207 Main Mall Vancouver, BC

V6T 1Z4, CANADA

E-MAIL: jim@stat.ubc.ca

Department of Mathematical Sciences

UNIVERSITY OF EXETER

Exeter, Devon

EX4 4SB UK

E-MAIL: G.Shaddick@exeter.ac.uk 